\title{
Entrenamiento vascular intensivo para el cirujano no vascular
}

\author{
Intensive vascular training for the non-vascular surgeon
}

Jorge L. Salazar-Alarcón ${ }^{1 *}$, María A. Valcárcel-Saldaña ${ }^{1}$, José G. Somocurcio-Vílchez', Alfredo L. Sotomayor-Estrada y Mónica Herencia-Souza ${ }^{3}$

${ }^{1}$ Departamento de Enseñanza, Instituto de Cirugía Experimental, Universidad Nacional Mayor de San Marcos, Lima; ${ }^{2}$ Departamento de Cirugía de Tórax y Cardiovascular, Hospital Nacional Hipólito Unanue, Lima; ${ }^{3}$ Departamento de Diagnóstico por Imágenes, Hospital Nacional Daniel Alcides Carrión, Bellavista. Perú

\section{Resumen}

Introducción: En la mayoría de los servicios de emergencia del Perú no se cuenta con un cirujano vascular, teniendo que resolver los problemas vasculares el cirujano general. Es necesario que los cirujanos generales estén entrenados en el manejo vascular, sobre todo de aquellas condiciones que pueden comprometer la vida. Objetivo: Compartir los resultados del primer curso de cirugía vascular para el cirujano no vascular y el nivel de satisfacción de los alumnos participantes. Método: El curso de entrenamiento utilizó cinco modelos de simulación distribuidos en cinco sesiones prácticas. Al final del curso se evaluó a los alumnos usando la escala OSATS (Objetive Structured Assesment of Technical Skills) y se midió el tiempo de anastomosis arterial; adicionalmente se evaluó el nivel de satisfacción del curso. Resultados: Participaron 12 alumnos, de los cuales ocho cumplieron los criterios de inclusión. La edad media fue de 39.9 años. La calificación OSATS fue de 19 ( \pm 2.1$)$. El tiempo de anastomosis fue de 17.6 ( \pm 7.9$)$ minutos. El nivel de satisfacción global de curso fue de 31.05 ; no hubo diferencias significativas entre la satisfacción de las diferentes sesiones prácticas $(p=0.85)$. El alfa de Cronbach de la encuesta fue de 0,96 (excelente). Conclusiones: El curso intensivo de entrenamiento vascular combina diferentes escenarios quirúrgicos basados en la simulación. El nivel de satisfacción del curso y la fiabilidad de la encuesta para «percepción de las prácticas de simulación» fueron excelentes.

Palabras clave: Curso básico. Cirugía vascular. Entrenamiento quirúrgico. Simulación. Educación médica.

\begin{abstract}
Introduction: In most emergency services in Peru there is no vascular surgeon, the general surgeon having to solve the vascular problems. General surgeons need to be trained in vascular management, especially those life-threatening conditions. Objective: To share the results of the first course of vascular surgery for the non-vascular surgeon and the level of satisfaction of the participating students. Method: The training course used five simulation models distributed in five practical sessions. At the end of the course, the students were evaluated using the OSATS (Objective Structured Assessment of Technical Skills) scale and the arterial anastomosis time was measured; Additionally, the level of satisfaction of the course was evaluated. Results: 12 students participated, 8 met the inclusion criteria, the mean age was 39.9 years, the OSATS score was 19 ( \pm 2.1 ) and the anastomosis time 17.6 ( \pm 7.9) minutes. The level of overall course satisfaction was 31.05 , there were no significant differences between the satisfaction of the different practical sessions $(p=0.85)$. The Cronbach's alpha for the survey was
\end{abstract}

\footnotetext{
Correspondencia:

*Jorge L. Salazar-Alarcón

Jr. Estados Unidos 297

Fecha de recepción: 04-04-2020

C.P. 15072, Jesús María, Lima, Perú $\quad$ Fecha de aceptación: 12-08-2020

E-mail: jorge.salazar@unmsm.edu.pe

DOI: $10.24875 / C I R U .20000267$ (http://creativecommons.org/licenses/by-nc-nd/4.0/).
} 
0.96 (Excellent). Conclusions: The intensive vascular training course combines different surgical scenarios based on simulation. The level of satisfaction of the course and the reliability of the survey "perception of simulation practices" were excellent.

Key words: Basic course. Vascular surgery. Surgical training. Simulation. Medical education.

\section{Introducción}

El aprendizaje de la cirugía sigue el modelo clásico de William Halsted, que consiste en mirar, hacer y enseñar un procedimiento quirúrgico. En los últimos años ha habido un debate intenso por buscar mejores herramientas de enseñanza-aprendizaje para la cirugía'. La simulación es una herramienta educativa que pone al aprendiz en un escenario que imita la realidad, lo que ha permitido el entrenamiento seguro para el paciente ${ }^{2}$. En diferentes partes del mundo se ofertan cursos de entrenamiento quirúrgico basado en simulación, que permiten la adquisición de habilidades y destrezas en cirugía tanto abierta como laparoscópica ${ }^{3}$.

Para el entrenamiento de la cirugía vascular se han utilizado modelos de impresiones tridimensionales, de cadáver y de animales, todos con resultados que impactan positivamente en el aprendizaje de los participantes $^{4,5}$. Sin embargo, la principal limitación de estos modelos es la falta de sangrado 6 . A pesar de ello, han demostrado proporcionar habilidades y destrezas iniciales o básicas en cirugía vascular ${ }^{6}$.

En el Perú, la cirugía vascular la practican los cirujanos de tórax y cardiovascular, y en menor medida de otras especialidades como parte de grupos muy especializados (cirugía de trasplante, cirugía oncológica y cirugía general). Sin embargo, las emergencias vasculares cada vez se vuelven más frecuentes, sea por trauma o incidentales, y son necesarios un correcto control y una adecuada reparación vascular, ya que el pronóstico dependerá del tratamiento otorgado ${ }^{7,8}$. A pesar de ello, en la mayoría de los servicios de emergencia del Perú, las emergencias vasculares son resueltas por el cirujano general o el ginecólogo de guardia.

El Instituto de Cirugía Experimental de la Universidad Nacional Mayor de San Marcos tiene una política de capacitación mediante la oferta de cursos curriculares y extracurriculares para el perfeccionamiento de habilidades y destrezas quirúrgicas; en tal sentido, se planificó y ejecutó un curso intensivo de entrenamiento en cirugía vascular para cirujanos no vasculares. El objetivo de este estudio es compartir los resultados de este curso y el nivel de satisfacción de los alumnos.

\section{Método}

Estudio de tipo cuasiexperimental. El curso tuvo una duración de 5 días y se desarrolló en cinco sesiones teóricas de conceptos básicos de técnica operatoria vascular y 10 horas de entrenamiento quirúrgico. El primer día de práctica se reconoció el instrumental vascular y se realizó sutura en un modelo de tela. El segundo día, el entrenamiento se realizó en arteria aorta de cabra (Capra aegagrus hircus) conservada en glutaraldehído al $2 \%$. El tercer día se suturó en una arteria de silicona. El cuarto día se realizó una anastomosis entre una arteria de silicona y una prótesis de poliéster (dacrón). El quinto día se disecó, seccionó y anastomosó una arteria femoral principal de cerdo (Sus scrofa domestica) (Fig. 1). Las sesiones teóricas y prácticas fueron impartidas por dos cirujanos vasculares expertos.

La obtención del modelo animal se realizó respetando la Declaración Universal de los Derechos de los Animales (UNESCO, 1989) y la Ley N. ${ }^{\circ} 27265$, Ley de protección a los animales domésticos y a los animales silvestres mantenidos en cautiverio, y la Ley de Protección y Bienestar animal 30220 vigentes en nuestro país.

Se realizó una evaluación al finalizar el curso mediante la escala OSATS (Objective Structured Assessment of Technical Skills) (Tabla 1). Además, se midió el tiempo en minutos para la realización de la anastomosis en la arteria femoral de cerdo. Para valorar la permeabilidad y la fuga de la anastomosis se conectó el cabo proximal de la arteria femoral a una sonda de alimentación n. ${ }^{0} 12$, la cual a su vez estaba conectada a cloruro de sodio teñido con aseptil rojo al 5\% . Se definió como fuga la mínima extravasación de cloruro de sodio a través de la sutura anastomótica, y como permeabilidad la ausencia de obstrucción a nivel de la anastomosis.

Una vez concluido el curso se aplicó una encuesta de satisfacción mediante un cuestionario de «Percepción de las prácticas de simulación». La encuesta 
Tabla 1. Escala OSATS para habilidades y destrezas quirúrgicas

\begin{tabular}{|c|c|c|c|c|c|}
\hline Puntuación & Respeto por los tejidos & $\begin{array}{l}\text { Tiempo y } \\
\text { movimientos }\end{array}$ & Uso del instrumento & $\begin{array}{l}\text { Flujo de la cirugía y } \\
\text { planificación posterior }\end{array}$ & Conocimiento \\
\hline 1 & $\begin{array}{l}\text { Uso frecuente de fuerza } \\
\text { innecesaria } \\
\text { Daño causado por uso } \\
\text { inapropiado de los } \\
\text { instrumentos }\end{array}$ & $\begin{array}{l}\text { Muchos } \\
\text { movimientos } \\
\text { innecesarios }\end{array}$ & $\begin{array}{l}\text { Repetidamente hace } \\
\text { movimientos vacilantes }\end{array}$ & $\begin{array}{l}\text { Con frecuencia detiene el } \\
\text { procedimiento o requiere } \\
\text { comentar los pasos } \\
\text { siguientes }\end{array}$ & $\begin{array}{l}\text { Conocimiento } \\
\text { deficiente y requiere } \\
\text { instrucciones en la } \\
\text { mayoría de los pasos } \\
\text { quirúrgicos }\end{array}$ \\
\hline 2 & - & - & - & - & - \\
\hline 3 & $\begin{array}{l}\text { Manejo cuidadoso de los } \\
\text { tejidos, pero ocasionalmente } \\
\text { produce daño inadvertido }\end{array}$ & $\begin{array}{l}\text { Relación } \\
\text { entre tiempo y } \\
\text { movimientos } \\
\text { eficiente, pero } \\
\text { realiza algunos } \\
\text { movimientos } \\
\text { innecesarios }\end{array}$ & $\begin{array}{l}\text { Uso competente de } \\
\text { los instrumentos, pero } \\
\text { ocasionalmente se } \\
\text { observa rígido o torpe }\end{array}$ & $\begin{array}{l}\text { Demuestra habilidades } \\
\text { para planear los pasos } \\
\text { siguientes, con progresión } \\
\text { constante del } \\
\text { procedimiento }\end{array}$ & $\begin{array}{l}\text { Conoce todos los } \\
\text { aspectos importantes } \\
\text { de la operación }\end{array}$ \\
\hline 4 & - & - & - & - & - \\
\hline 5 & $\begin{array}{l}\text { Maneja los tejidos } \\
\text { apropiadamente con mínimo } \\
\text { daño }\end{array}$ & $\begin{array}{l}\text { Economía de } \\
\text { movimientos y } \\
\text { máxima eficiencia }\end{array}$ & $\begin{array}{l}\text { Movimientos fluidos con } \\
\text { los instrumentos y sin } \\
\text { a torpeza }\end{array}$ & $\begin{array}{l}\text { Curso planeado de la } \\
\text { operación en forma obvia, } \\
\text { con flujo sin esfuerzo }\end{array}$ & $\begin{array}{l}\text { Familiaridad } \\
\text { demostrada con } \\
\text { todos los aspectos de } \\
\text { la operación }\end{array}$ \\
\hline
\end{tabular}
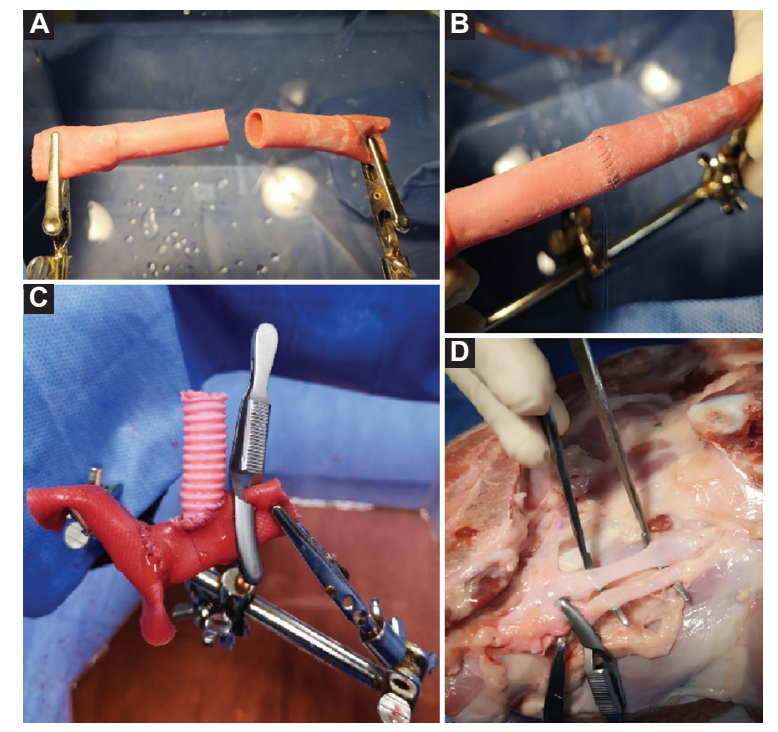

Figura 1. A y B: modelos de simulación, anastomosis en arteria de silicona. C: anastomosis silicona-dacrón. D: disección de arteria femoral de cerdo.

incluye siete aseveraciones y cada una de ellas se puede responder según una escala de Likert de 5 puntos (1: completamente en desacuerdo; 2 : desacuerdo; 3: no está seguro o no tiene opinión, 4: de acuerdo; 5: completamente de acuerdo), obteniendo un puntaje mínimo de 7 y máximo de 35 en cuanto al grado de satisfacción ${ }^{2}$.

\section{Criterios de inclusión y exclusión}

Los criterios de inclusión fueron ser médico cirujano con especialidad quirúrgica no vascular, estar inscrito en el curso de entrenamiento vascular, haber asistido a todas las sesiones teóricas y prácticas, y firmar el consentimiento informado de participación en el estudio. Se excluyó a cuatro alumnos que no completaron todas las sesiones de entrenamiento.

\section{Estadística}

Los datos recogidos se ingresaron y tabularon en una base de datos Excel versión 2010. El análisis incluyó medidas de frecuencia media, desviación estándar y porcentajes para la puntuación OSATS, tiempo de anastomosis, fuga y permeabilidad anastomótica. Se calculó el punto de corte Angoff para el OSATS global y el coeficiente intraclase para evaluar la concordancia entre los evaluadores al aplicar la escala OSATS.

Sobre la satisfacción del curso se calculó el promedio para cada aseveración del cuestionario, y además se halló el promedio global de cada práctica y de todo el curso con su respectiva desviación estándar e intervalo de confianza. Se realizó el análisis de normalidad con la prueba de Shapiro-Wilk para los promedios globales de cada práctica. Debido a que los puntajes totales no seguían una distribución normal, se realizó 
Tabla 2. Características de los alumnos asistentes al curso intensivo de entrenamiento vascular para cirujanos no vasculares

\begin{tabular}{|c|c|}
\hline Característica & \\
\hline Edad, años (media $\pm D E$ ) & $39.9 \pm 10.6$ \\
\hline \multicolumn{2}{|l|}{ Sexo, n (\%) } \\
\hline Femenino & $1(12.5)$ \\
\hline Masculino & $7(87.5)$ \\
\hline \multicolumn{2}{|l|}{ Especialidad, n (\%) } \\
\hline Cirugía general & $6(75)$ \\
\hline Urología & $1(12.5)$ \\
\hline Ginecología & $1(12.5)$ \\
\hline \multicolumn{2}{|l|}{ Institución de Trabajo, n (\%) } \\
\hline EsSalud & $6(75)$ \\
\hline MINSA & $2(25)$ \\
\hline \multicolumn{2}{|l|}{ Procedencia, n (\%) } \\
\hline Lima & $6(75)$ \\
\hline Abancay & $1(12.5)$ \\
\hline Cuzco & $1(12.5)$ \\
\hline \multicolumn{2}{|c|}{ Suturas vasculares previas, n (\%) } \\
\hline Ninguna & $4(50)$ \\
\hline Una & $1(12.5)$ \\
\hline Dos & $3(37.5)$ \\
\hline \multicolumn{2}{|c|}{ Anastomosis vasculares previas, n (\%) } \\
\hline Ninguna & $7(12.5)$ \\
\hline Una & $1(87.5)$ \\
\hline
\end{tabular}

un análisis no paramétrico mediante la prueba de Kruskal-Wallis para evaluar si había diferencia entre los promedios de percepción de las prácticas. Adicionalmente se estimó el coeficiente alfa de Cronbach de la encuesta de satisfacción.

\section{Aspectos éticos}

La encuesta se realizó previo consentimiento informado de los alumnos, de forma anónima y respetando la confidencialidad de los datos. De igual manera, la información brindada se usó únicamente con fines académicos y solo fue manejada por los investigadores.

\section{Resultados}

En el curso de entrenamiento vascular intensivo para el cirujano no vascular participaron 12 alumnos, de los cuales ocho cumplieron los criterios de inclusión para ser considerados en el estudio (Tabla 2). Ninguno de los alumnos había realizado entrenamiento vascular previo. El mayor puntaje obtenido aplicando la escala OSATS fue de 21 y el menor tiempo para realizar la anastomosis arterial termino-terminal fue
Tabla 3. Resultados de la evaluación final del curso intensivo de entrenamiento vascular para cirujanos no vasculares

\begin{tabular}{lc}
\hline Resultado & Valor \\
\hline OSATS, media \pm DE & $19 \pm 2.1$ \\
Tiempo de anastomosis, minutos, & $17.6 \pm 7.9$ \\
media \pm DE & \\
Fuga anastomótica, n (\%) & $1(12.5 \%)$ \\
Sí & $7(87.5 \%)$ \\
No & \\
Permeabilidad de la anastomosis, n (\%) & $7(87.5 \%)$ \\
Completa & $1(12.5 \%)$ \\
\hline Parcial &
\end{tabular}

de 11 minutos. Tres alumnos (37.5\%) lograron nota aprobatoria en la escala OSATS (21 puntos) (Tabla 3 ). El punto de corte Angoff para la puntuación OSATS global se estableció en 21 puntos (de 25, equivalente a 84\%). El coeficiente de correlación intraclase entre evaluadores fue de 0.82 (casi perfecto).

El grado de satisfacción del curso fue de 31.05 (88.7\%); todas las prácticas tuvieron un promedio (media) de calificación $>30$. La práctica del quinto día, disección y anastomosis en arteria de cerdo, fue la que obtuvo la mayor satisfacción (Tabla 4). No hubo diferencias significativas entre el grado de satisfacción con las diferentes prácticas de entrenamiento $(p=0.85)$ (Tabla 5). El alfa de Cronbach obtenido para el total de las preguntas de la encuesta de satisfacción fue de 0.96 (excelente).

\section{Discusión}

Este estudio se realizó con el objetivo de compartir los resultados del curso intensivo de entrenamiento vascular para cirujanos no vasculares y evaluar el grado de satisfacción de los alumnos.

El curso se caracteriza por ser intensivo y secuencial; intensivo, porque concentra un gran volumen de entrenamiento en pocos días, y secuencial porque está constituido por diferentes escenarios de simulación quirúrgica que distribuidos cronológicamente buscan mejorar el proceso enseñanza-aprendizaje de la cirugía vascular, yendo de menor a mayor grado de complejidad. Con respecto a las habilidades y destrezas medidas al final del curso con la escala OSATS, nuestros alumnos obtuvieron una media general por debajo de la necesaria para aprobar el curso (19 de 21puntos); sin embargo, al disgregar estos resultados observamos que tres 
Tabla 4. Resultados de la encuesta de percepción de las prácticas de simulación del curso intensivo de entrenamiento vascular para cirujanos no vasculares

\begin{tabular}{|c|c|c|c|c|c|c|}
\hline $\mathrm{N} .^{\circ}$ & Aseveración & $\begin{array}{c}\text { Práctica } \\
1^{*}\end{array}$ & $\begin{array}{l}\text { Práctica } \\
2^{*}\end{array}$ & $\begin{array}{l}\text { Práctica } \\
3^{*}\end{array}$ & $\begin{array}{l}\text { Práctica } \\
4^{*}\end{array}$ & $\begin{array}{l}\text { Práctica } \\
5^{\star}\end{array}$ \\
\hline 1 & $\begin{array}{l}\text { La práctica de modelos simulados mejora mis destrezas } \\
\text { relacionadas con la realización de este procedimiento }\end{array}$ & 4.6 & 4.5 & 4.4 & 4.5 & 4.6 \\
\hline 2 & $\begin{array}{l}\text { Recibí retroalimentación educacional útil en la (s) } \\
\text { sesión (es) de entrenamiento }\end{array}$ & 4.4 & 4.4 & 4.3 & 4.4 & 4.4 \\
\hline 3 & $\begin{array}{l}\text { La práctica con el modelo permite cometer errores que } \\
\text { probablemente sucedan en el ambiente clínico real }\end{array}$ & 4.5 & 4.5 & 4.4 & 4.5 & 4.5 \\
\hline 4 & $\begin{array}{l}\text { El modelo usado en el taller simula o representa el } \\
\text { procedimiento de manera realista }\end{array}$ & 3.9 & 4.1 & 4.4 & 4.5 & 4.5 \\
\hline 5 & $\begin{array}{l}\text { La práctica de procedimientos con el modelo simulado } \\
\text { refuerza mi confianza en relación con mis destrezas } \\
\text { clínicas }\end{array}$ & 4.4 & 4.5 & 4.5 & 4.4 & 4.5 \\
\hline 6 & $\begin{array}{l}\text { Los talleres prácticos, usando modelos de procedimientos } \\
\text { simulados, deberían ser un componente obligatorio en la } \\
\text { enseñanza de la medicina }\end{array}$ & 4.5 & 4.5 & 4.5 & 4.5 & 4.5 \\
\hline 7 & $\begin{array}{l}\text { La práctica con modelos de simulación me ayuda a } \\
\text { prepararme para realizar procedimientos clínicos de mejor } \\
\text { manera que solo con la experiencia clínica exclusiva }\end{array}$ & 4.6 & 4.4 & 4.4 & 4.5 & 4.5 \\
\hline
\end{tabular}

Tabla 5. Grado de satisfacción del curso intensivo de entrenamiento vascular para cirujanos no vasculares usando la encuesta de percepción de las prácticas de simulación

\begin{tabular}{|c|c|c|c|}
\hline Práctica de simulación & Media ( \pm DE) & IC & $\mathbf{p}^{*}$ \\
\hline Anastomosis en arteria de tela & $30.88( \pm 3.7)$ & 27.79-33.95 & \\
\hline Rafia y anastomosis en arteria de cerdo conservada & $30.88( \pm 3.9)$ & $27.57-34.17$ & \\
\hline Anastomosis en arteria de silicona & $30.75( \pm 4.5)$ & 27.01-34.48 & 0.85 \\
\hline Anastomosis de silicona-dacrón & $31.25( \pm 4.0)$ & $27.01-34.48$ & \\
\hline Disección y anastomosis de arteria femoral de cerdo & $31.51( \pm 3.5)$ & 27.88-34.61 & \\
\hline Satisfacción global & $31.05( \pm 3.92)$ & $29.85-32.24$ & \\
\hline
\end{tabular}

DE: desviación estándar; IC: intervalo de confianza.

*Prueba de Kruskal-Wallis.

alumnos sí lograron aprobar. Estos resultados podrían explicarse en cierta medida debido a que la evaluación final exige contar con habilidades y destrezas avanzadas; no obstante, los cursos intensivos han mostrado mayor efectividad en la adquisición de habilidades básicas o simples ${ }^{10}$. Consideramos que, a pesar de ello, los resultados son satisfactorios, ya que la ausencia de fuga y la permeabilidad completa de la anastomosis se logró en el $87.5 \%$ de los casos, puesto que si bien la escala OSATS valora el desempeño del alumno mostrado en el respeto por los tejidos, el tiempo y la calidad de los movimientos, el uso de los instrumentos, la planificación del procedimiento y el conocimiento de la técnica, no valora el resultado final (anastomosis). Cabe mencionar que existen numerosas herramientas para evaluar las habilidades y destrezas quirúrgicas; no obstante, tal como reportan Vaidya, et al. ${ }^{11}$, la más utilizada es la OSATS a pesar de que no está exenta de cuestionamientos, lo que coincide con lo ya expuesto.

En relación con el tiempo para realizar la anastomosis arterial, si bien nuestro trabajo no nos permite evaluar el impacto del entrenamiento en términos de 
tiempo, consideramos que es un tiempo aceptable según lo reportado por Wilhelm, et al..$^{12}$, quienes tras un curso intensivo de cirugía vascular de 2.5 días encontraron que los asistentes mejoraron significativamente el tiempo necesario para la sutura venosa en 5.4 minutos (de 19.5 a 14.1 minutos) al final del entrenamiento. Del mismo modo, Varas, et al. ${ }^{13}$ reportaron que, en un programa de 16 días de entrenamiento de habilidades laparoscópicas básicas para la realización de tres nudos intracorpóreos, se logró un descenso gradual del tiempo durante los primeros 5 días de entrenamiento y de ahí en adelante el tiempo no se modificó significativamente. Por tanto, se infiere que el curso intensivo tiene un impacto positivo que deberá ser comprobado en un estudio futuro.

La simulación ha ido evolucionado hasta convertirse hoy en día en una herramienta inobjetable de la capacitación quirúrgica. Dentro de sus beneficios se reportan la mejora en la confianza de los cirujanos, el aumento de la autonomía intraoperatoria de los residentes, la transferencia de habilidades al quirófano, la reducción del tiempo operatorio y la mejora de la calidad de los procedimientos, entre otros ${ }^{14-18}$. Si bien los beneficios son amplios, surge la pregunta de por qué no se aplica universalmente. Algunos autores citan que la principal causa es la falta de recursos y de fondos para su implementación ${ }^{3,19}$. Al ser nuestro curso netamente simulado, consideramos esencial evaluar el grado de satisfacción mediante la medición del clima de aprendizaje, definido como la percepción de las características formales e informales de la educación que se está recibiendo. En nuestro caso no hallamos diferencias significativas entre el grado de satisfacción con las diferentes prácticas. Esto contrasta con lo hallado por Duran, et al. ${ }^{20}$, quienes reportan que los residentes de quinto año a más preferían modelos de disección en cadáver y entrenamiento endovascular, y por el contrario los residentes de primero a cuarto año preferían el modelo anastomótico, pero el $86 \%$ de los residentes encuestados creía que la simulación tenía un papel educativo importante en el proceso de enseñanza-aprendizaje. Estos hallazgos contradictorios se podrían explicar por la mayor apreciación crítica que tiene un residente de cirugía vascular al estar inmerso en la especialidad, en comparación con nuestros alumnos, que son cirujanos no vasculares con casi nula experiencia en la materia. En términos generales, nuestro curso obtuvo un excelente grado de satisfacción, lo que concuerda con la percepción positiva hacia la simulación de los alumnos del estudio de Duran, et al. ${ }^{20}$ antes mencionado.
Finalmente, es necesario señalar que este estudio solo describe el resultado final de la implementación y la ejecución de un curso de entrenamiento intensivo, y no puede realizarse un mayor análisis debido a que no se cuenta con evaluaciones anteriores al entrenamiento ni con grupo control (expertos); además, somos conscientes de que el pequeño tamaño muestral podría conllevar errores de interpretación de los resultados.

\section{Conclusiones}

El curso intensivo de entrenamiento vascular combina diferentes escenarios quirúrgicos basados en la simulación. El grado de satisfacción con el curso fue excelente. Es necesario demostrar la efectividad del curso en futuros estudios.

\section{Financiamiento}

Propio de los investigadores.

\section{Conflicto de intereses}

Los autores declaran no tener ningún conflicto de intereses.

\section{Responsabilidades éticas}

Protección de personas y animales. Los autores declaran que los procedimientos seguidos se conformaron a las normas éticas del comité de experimentación humana responsable y de acuerdo con la Asociación Médica Mundial y la Declaración de Helsinki.

Confidencialidad de los datos. Los autores declaran que han seguido los protocolos de su centro de trabajo sobre la publicación de datos de sujetos referidos.

Derecho a la privacidad y consentimiento informado. Los autores han obtenido el consentimiento informado de los pacientes y/o sujetos referidos en el artículo. Este documento obra en poder del autor de correspondencia.

\section{Bibliografía}

1. Costa GOF da, Rocha HAL, Moura Júnior LG de, Medeiros F das C. Taxonomy of educational objectives and learning theories in the training of laparoscopic surgical techniques in a simulation environment. Rev Col Bras Cir. 2018;45:e1954.

2. Villagrán I, Tejos R, Chahuan J, Uslar T, Pizarro M, Varas J, et al. Percepción de estudiantes de pregrado de medicina de talleres de simulación de procedimientos médico-quirúrgicos. Rev Med Chile. 2018;146:786-95.

3. Robertson V, Davies R. Provision of simulation-based training (SBT) within UK vascular surgery training programmes. Surgeon. 2019;17:321-5. 
4. James HK, Chapman AW, Pattison GTR, Griffin DR, Fisher JD. Systematic review of the current status of cadaveric simulation for surgical training. Br J Surg. 2019;106:1726-34.

5. Achurra P, Mondragón G, Caro I, Figueroa D, Marine L, Mery D, et al Modelo pionero de entrenamiento en trauma vascular impreso en $3 \mathrm{D}$ en base a imágenes de pacientes reales: un trabajo interdisciplinario de simulación en educación quirúrgica. Inv Ed Med. 2017;6:133-4.

6. Sierra-Juárez MA, Cruz-Romero $\mathrm{Cl}$, Godinez-Vidal AR, Durán-Padilla MA Programa de entrenamiento en reparacion vascular en modelo experimental para residentes de cirugía general. Cir Cir. 2018;86:1230.

7. Tyburski JG, Wilson RF, Dente C, Steffes C, Carlin AM. Factors affecting mortality rates in patients with abdominal vascular injuries. J Trauma. 2001;50:1020-6.

8. Siufi Neto J, Santos Siufi DF, Magrina JF. Trocar in conventional laparoscopic and robotic-assisted surgery as a major cause of iatrogenic trauma to the patient. Best Pract Res Clin Obstet Gynaecol. 2016;35:13-9.

9. Martin JA, Regehr G, Reznick R, Macrae H, Murnaghan J, Hutchison C et al. Objective structured assessment of technical skill (OSATS) for surgical residents. Br J Surg. 1997;84:273-8.

10. Morales-García D, Alcázar-Montero JA, Sanz-Sánchez M, Miguelena-Bobadilla JM. La simulación como modelo de enseñanza en cirugía. Ci Esp. 2018:96:313-4.

11. Vaidya A, Aydin A, Ridgley J, Raison N, Dasgupta P, Ahmed K. Current status of technical skills assessment tools in surgery: a systematic review. J Surg Res. 2020;246:342-78.

12. Wilhelm M, Klemm K, Assadian A, Schmidli J, Schumacher H, Merrelaar $\mathrm{J}$, et al. Besser und schneller!: Evaluation eines 2,5-tägigen Gefäßchirurgischen Grundkurses für ChirurgInnen in Weiterbildung. Chirurg. 2013;84:125-9.
13. Varas J, Mejía R, Riquelme A, Maluenda F, Buckel E, Salinas J, et al. Significant transfer of surgical skills obtained with an advanced laparoscopic training program to a laparoscopic jejunojejunostomy in a live porcine model: feasibility of learning advanced laparoscopy in a general surgery residency. Surg Endosc. 2012;26:3486-94.

14. Robinson WP, Doucet DR, Simons JP, Wyman A, Aiello FA, Arous E, et al. An intensive vascular surgical skills and simulation course for vascular trainees improves procedural knowledge and self-rated procedural competence. J Vasc Surg. 2017;65:907-915.e3.

15. Kim SC, Fisher JG, Delman KA, Hinman JM, Srinivasan JK Cadaver-based simulation increases resident confidence, initial exposure to fundamental techniques, and may augment operative autonomy. J Surg Educ. 2016;73:e33-41.

16. James HK, Chapman AW, Pattison GTR, Griffin DR, Fisher JD. Systematic review of the current status of cadaveric simulation for surgical training. Br J Surg. 2019;106:1726-34.

17. Egle JP, Malladi SVS, Gopinath N, Mittal VK. Simulation training improves resident performance in hand-sewn vascular and bowel anastomoses. J Surg Educ. 2015;72:291-6.

18. Sidhu RS, Park J, Brydges R, MacRae HM, Dubrowski A. Laboratory-based vascular anastomosis training: a randomized controlled trial evaluating the effects of bench model fidelity and level of training on skill acquisition. J Vasc Surg. 2007;45:343-9.

19. Moreira RCR. Critical issues in vascular surgery: education in Brazil. $J$ Vasc Surg. 2008:48:87S-89S.

20. Duran C, Bismuth J, Mitchell E. A nationwide survey of vascular surgery trainees reveals trends in operative experience, confidence, and attitudes about simulation. J Vasc Surg. 2013;58:524-8. 